\title{
A Survey on Blockchain and Internet of Things
}

\author{
Tanweer Alam
}

\begin{abstract}
In the age of next-generation computer, the role of the cloud, the internet and smart devices will become stronger. These days we all know the word smart well. This word is often used in our daily lives. The Internet of Things (IoT) will generate a variety of information from a variety of resources. It can store big data in the cloud. Fog computing acts as a signal between cloud and IoT. Fog extensions in this framework apply to material under IoT. IoT devices are called Fog nodes, which can be accessed anywhere within the network range. A blockchain is a novel way of recording in a secure sequence. Creating a new framework in the development of Internet of Things is one of the critical problems of wireless communication where solving such a problem can lead to continued growth in the use and popularity of IoT. Proposed research creates a framework for providing a framework for middleware on the internet of smart devices network for the internet of things using blockchains technology. Our great offering connects new research that integrates blockchains into the Internet of Things and provides secure Internet connection for smart devices. Blockchain (BC) Internet of Things (IoT) is a new technology that works with low-level, distributed, public and real-time leaders to maintain transactions between IoT sites. A blockchain is a series of blocks, each block being linked to its previous blocks. All blocks have cryptographic hash code, previous block hash, and its data. Transactions in BC are the basic components used to transfer data between IoT nodes. IoT nodes are a variety of portable but smart devices with embedded sensors, actuators, systems and the ability to communicate with other IoT nodes. The role of BC in IoT is to provide a process for processing secure data records using IoT nodes. $\mathrm{BC}$ is a protected technology that can be used publicly and openly. IoT requires this type of technology to allow secure communication between IoT nodes in different environments. Events in BC can be tracked and monitored by anyone who is certified to communicate within IoT.
\end{abstract}

Index Terms-Blockchain, Internet of Things, Security, Privacy, Wireless Communication.

\section{INTRODUCTION}

$\mathrm{T}$ HE IoT is growing exponentially year by year with its focus on $5 \mathrm{G}$ technologies, such as Smart Homes and Cities, Health, intelligence surveillance etc. But there are challenges to security and privacy. IoT devices are connected in a shared power. Therefore, it is very complicated to use the most common security methods available for communication between IoT nodes. The proposed research is a step forward in

Tanweer Alam is with faculty of Computer and Information Systems, Islamic University of Madinah, Madinah, Saudi Arabia. Email: tanweer03@iu.edu.sa wireless communication with IoT where we propose a new middleware framework based on blockchain technology. Wireless communication is the Internet key for things. It is expected to exceed 50 billion connected devices by 2020 and most of these nodes cannot be connected via wireline. In order to enable critical systems such as intelligent industries or intelligent structures, communication processes must address the ambiguous nature of wireless links. The research work proposed in this project is to develop and implement a middleware framework based on blockchain technology in the construction of the Internet of Things. The result of the research is to establish a new IoT framework. The proposed research uses the correct and effective imitation of the study you are looking for and can be done through the Internet of Things. In the future, researchers could expand this research and use it online for everything. Creating a new middleware-based block ware framework in the development of Internet of Things can be an important framework for improving the performance of the IoT framework in a unique environment. Wireless communication is a fast-growing research area that enables users to interact without using cables. Internet of Things is based on a completely wireless network. At the beginning of the Internet, it was developed to communicate from one device to another using access to browsers. However, in the modern era, high-performance high-speed devices have many advanced technologies such as low power consumption etc. Available to communicate with others. Fog extensions in this framework apply to material under IoT. IoT devices are called Fog nodes, which can be accessed anywhere within the network range. This research will help the IoT framework. However, the analysis framework is studied in the literature review, the authors did not describe the full framework in this study. This study adds a blockchain and an advanced fog to improve the effective IoT framework for communication between smart devices. Comparisons of this study with the limitations of the reexamination of previous research, admissions, bulk variations and poor distribution of production packages are mandatory. The algorithm was used for testing. The proposed framework accurately predicts our comprehensive assessment. In addition, confirm the results of the statistics. The purpose of this study is to create a new model of communication between the Internet of Things and Fog computing. This research is based on blockchain technology with Middleware, Fog, and IoT. The main contribution of this study is to design an Internet 
communication framework using fog and blockchain technology. The proposed framework is specifically suited for applications where data is periodically transferred to the natural network of smart devices. In these applications, on the other hand, packets are produced based on a specific time pattern. On the other hand, service time is constantly changing randomly with standard distribution. Therefore, the service time may be temporarily delayed, as an inevitable result some packages may encounter a busy channel and be discarded. We solve this problem by proposing a new middleware framework. We show that the proposed IoT-Fog framework, not only increases inputs, but also direct connections between generations (sensors) and communication packet systems are removed which makes the system more stable. In addition, in order to improve the proposed model, we have hired a redistribution system, flexible pack length, and full vehicle condition. The solution to this study is summarized as follows. The implementation of the IoT-Fog framework for internet connectivity for $5 \mathrm{G}$ smart devices will be set to work online for things. The concept will work with a three-layer model, these layers are Fog, Blockchain and IoT. The proposed research supports wireless communication technology to establish the IoT-Fog framework within the network of device devices.

The proposed framework introduces and presents its role in IoT. The IoT-Fog framework has the following features:
a) Devices (Items)
b) The Internet
c) Middleware
d) Using fog with blockchain

In fog, status servers store secure resources, proxies by thirdparty servers can store protected data and owners are legitimate devices. The key server in middleware creates encryption keys. A token given to a smart device by an authorized blockchains database has the authority to access the framework, request keys from the key server, and download data to the cloud. Figure 1 presents the components of the proposed framework.

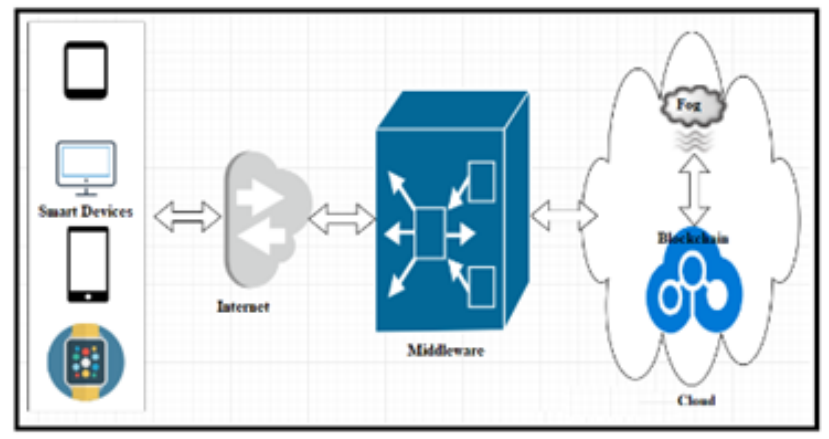

FIGURE 1. IoT-Fog Framework

The The following steps are applied to the proposed framework.

1. Smart contracts published by status servers, attorneys and fog owners in an authorized database of blockchains.

2. IoT smart device detects smart contacts from authorized database of blockchains.

3. An authorized blockchains database archive creates an intelligent IoT device token.
4. The smart device asks for the keys from the key server in the middleware and sends the token with the application.

5. Key server in middleware verify the token from the authorized blockchain database and create a smart device key and feedback back to the smart device.

6. Now the smart IoT device is authorized to access data from the cloud.

The framework itself is divided into three layers: IoT device layer, Fog layer, and cloud layer.

The framework can provide QoS by reducing traffic congestion and variability in the number of smart devices. In this study, we look at the state of inactivity in order to make our tests more effective, at which point, the general performance regarding the overall performance of the framework is assessed. IoT-Fog in this framework will view and analyze real-time data collected on fog nodes and take action.

The following are the key points-

1. The study is mainly focused on IoT. Enables smart devices to communicate with another device within the Internet of smart devices using blockchain technology.

2. The proposed communication framework will access the internet for smart devices.

3. The results of the proposed study will be compared with the previous study in the same area.

\section{BACKGROUND}

In 1991, Theodore S. Rappaport published an article entitled "The Wireless Revolution", in which he introduced wireless communication technology as the key to communication between people as well as devices. In 1994, Andy Harter and Andy Hopper published an article entitled "A Distributed Location System for the Active Office", in this paper, outlining infrared sensor arrangements using communication badges between devices and workstations. In 1994, Tristan Richardson, Frazer Bennett, Glenford Mapp, and Andy Hopper presented the article "A ubiquitous, personalized computer Telephony in the X Window System Environment", in this article they introduced $\mathrm{X}$ windows, $\mathrm{X}$ programs protocol for securing communication between client and server. In the article, the authors represented the "System Software for Ubiquitous Computing" for the integration of different types of networks, and created connections between devices on different types of networks. In 2002 the researchers published an article entitled "Connecting the Physical World with Pervasive Networks", in which they addressed the challenges and opportunities of using the physical world through widespread compte-rich computation networks. Cloud computing came as a result of the continued development of computer paradigms. The advent of this technology has created the emergence of software (SaaS) as a service that says consumers do not need to buy software rather than go according to their needs. In 2006, Amazon achieved a milestone by testing the elastic elastic computing cloud (EC 2) that started the computer. * However, the term cloud computing did not coincide until March 2007. The following year saw the rapid development of this new system. In addition, cloud infrastructure services have expanded to 
install software (SaaS) as a service. In mid-2012, Oracle's cloud was introduced, which supports a wide range of deployments. For example, it could lead to more than 139,000,000 games on Google In May 2014, Lihong Jiang et al published an article entitled "An IoT-Oriented Data Storage Framework in Cloud Computing Platform", focusing on the framework storage of relevant data allow not only to properly store large IoT data, but also to integrate both scheduled unstructured data. In this article, the IoT biological system and key technologies to support IoT communication are introduced. In 2016, Maria Rita Palattella et al published an article entitled "Internet of Things in the 5G Era: Enablers, Architecture and Business Models", in this article they introduced IoT 5G technology, exploring both technological aspects and standards. In 2018, Pradip Kumar Sharma, Yen Chen and Jong Hyuk Par published an article entitled, "Software Defined Fog Node Based Distributed Blockchain Cloud Architecture IoT". They introduced the software-defined environment using the IoT blockchain cloud.

\section{RESEARCH IMPORTANCE}

Building a new reliable framework based on IEEE 802.15.4 online communication for smart devices can be an important framework for improving the reliability of communication. Wireless networks enable users to interact with each other in the IoT environment. But there are many challenges to secure and reliable communication. Early in the Internet, It was developed to connect one device to another device using browser access. However, in the modern era, high-performance devices have many advanced technologies such as low power consumption etc. Available to communicate with others. Communication reliability has been suggested as one of the most important issues for wireless communication where solving such a problem can lead to continued growth in IoT use and popularity. The proposed research creates a framework to provide internet connectivity for the network of smart devices for the internet of things using IEEE802.15.4. Our great offering connects new research that combines reliability in the Internet of Things and provides reliable online connectivity for smart devices. This research will help the IoT framework. However, the analytical framework is read in book reviews, the authors did not describe the full framework in their essays. This study adds an improved Markov-chain and MAC framework to improve the effective framework of communication between smart devices. Comparisons of this study with the limitations of the re-examination of previous research, validation, bulk variability and poor distribution of production packages are mandatory. The algorithm was used for testing. The proposed framework accurately predicts our comprehensive assessment. In addition, the imitation of Monte-Carlo confirms the mathematical results. Smart devices are growing increasingly day by day around the world. They offer a lot of services to end users and attach to their daily lives. Smart devices can easily connect to the Internet by sending and receiving data within a network. Smart devices are not just Smartphones, it can be a smart refrigerator, Smart home entry, smart air conditioners, Smart hubs, Smart thermostat, Color changing LED smartphones, Smart Watches and tablets smart etc in the online framework of things, connected to each other via the internet.

TABLE 1: IoT Devices installed category and year wise (in Millions)

\begin{tabular}{|c|c|c|c|c|}
\hline $\begin{array}{l}\text { Categor } \\
\mathrm{y}\end{array}$ & 2016 & 2017 & 2018 & 2020 \\
\hline $\begin{array}{c}\text { IoT } \\
\text { Devices }\end{array}$ & ${ }_{0} 3963$. & $3^{5244 .}$ & 7036.3 & $0_{0} 12863$. \\
\hline $\begin{array}{l}\text { Business } \\
: \quad \text { Across } \\
\text { Industries }\end{array}$ & $1^{1102 .}$ & $0^{1501 .}$ & 2132.6 & 4381.4 \\
\hline $\begin{aligned} & \text { Business } \\
&: \quad \text { Vertical } \\
& \text { specific }\end{aligned}$ & $6^{1316 .}$ & $4^{1635 .}$ & 2027.7 & 3171.0 \\
\hline Total & $8^{6381 .}$ & $6^{8380 .}$ & $6^{11196 .}$ & $4^{20415 .}$ \\
\hline
\end{tabular}

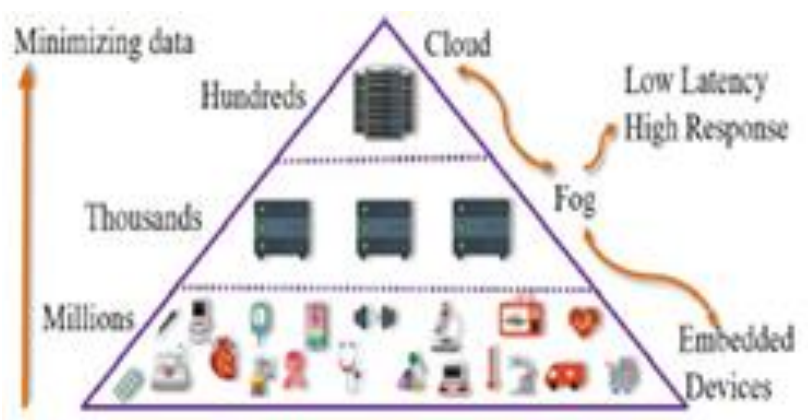

FIGURE 2. Cloud, Fog and embedded devices

The proposed research program creates research to facilitate online communication of objects using fog technology and blockchain. Transferring data from one configuration to another using a wireless network dates back to 1973 in the form of radio network packets. They were able to communicate with other similar configuration devices. Recent work is underway on a project called Serval Project. Provides networking location for Android communication devices on a sub-network. While our research is concerned with the online connection of objects. The main contribution of this study is the construction of a communication framework and provide reliable and fast communication using fog and blockchain between the internet of smart devices. Previous studies have focused on building and utilizing a communication framework, but such research does not create a complete framework for IoT-Fog communication between the internet of smart devices.

$\mathrm{BC}$ is a technology that provides transaction security between IoT devices. Provides shared, distributed and publicly available shared leagues to store used blockchain data and authentication on the IoT network. Information stored in a public ledger is automatically managed using a peer-to-peer topology. $\mathrm{BC}$ is a technology in which transactions are drawn in the form of a block in BC between IoT nodes. Blocks are connected and all devices have the device's previous address. Blockchain and IoT together work in the framework of IoT and Cloud integration. In the future, $\mathrm{BC}$ will alter IoT interactions. The objectives for the integration of $\mathrm{BC}$ and IoT can be 
summarized as follows.

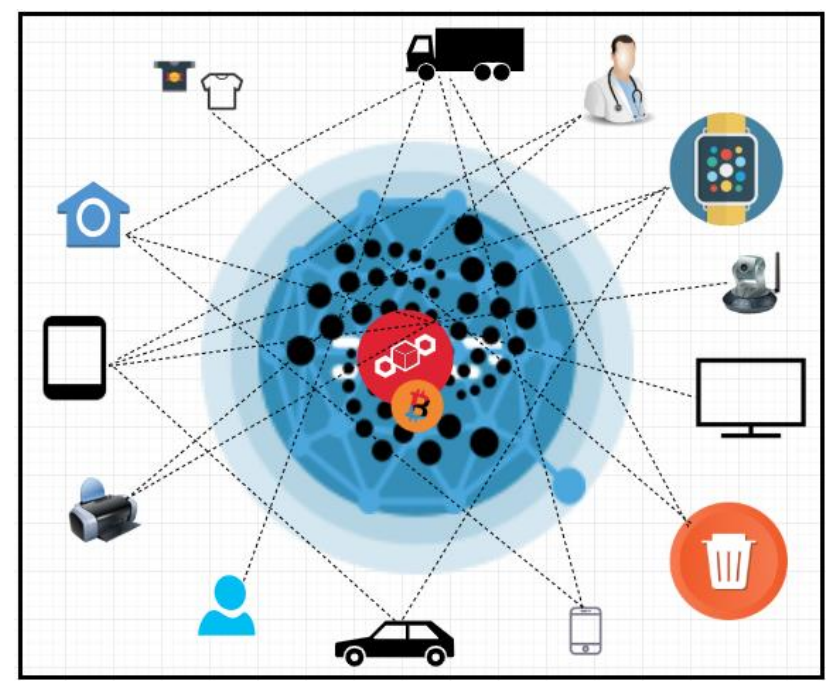

Figure 3 : Blockchains and IoT

i) Distributed framework: This method is similar to IoT and BC. It is removed from one system and provide location for the program in the field. It improves the chances of failure and performance of the entire system.

ii) Security: In BC, transactions between nodes are secure. It is a novel way of secure communication. BC allows IoT devices to communicate securely.

iii) Identification: In IoT, all connected devices are identified separately with a unique ID. All BC blocks are also identified separately. Therefore, $\mathrm{BC}$ is a reliable technology that provides specially identified information stored in public records.

iv) Reliability: IoT sites in $\mathrm{BC}$ have the ability to verify information transmitted over a network. The details are reliable because they were verified by the miners before entering $\mathrm{BC}$. Only verified blocks can enter BC.

v) Independence: In BC, all IoT nodes are free to connect to any node in a network without an intermediate system.

vi) Variety: In BC, IoT devices will communicate widely available, a distributed intelligence network that connects to the destination device in real time and with exchange details.

The other paper is summarized as follows: section 1 represents the presentation of the paper, section 2 represents the literature review, section 3 introduces the role of BC in IoT, section 4 represents the possibilities of an integrated approach, section 5 represents the challenges and section 6 represents the conclusion.

\section{Methods}

Security and privacy in communication between IoT devices are of paramount importance in 2020 and 2021. The authors introduced "IoTChain" to verify the information exchanged between the two sites in the IoT network. They introduced an algorithm for exchanging data on IoT and blockchains. In this paper, the authors focus on the security component of the IoTChain framework.

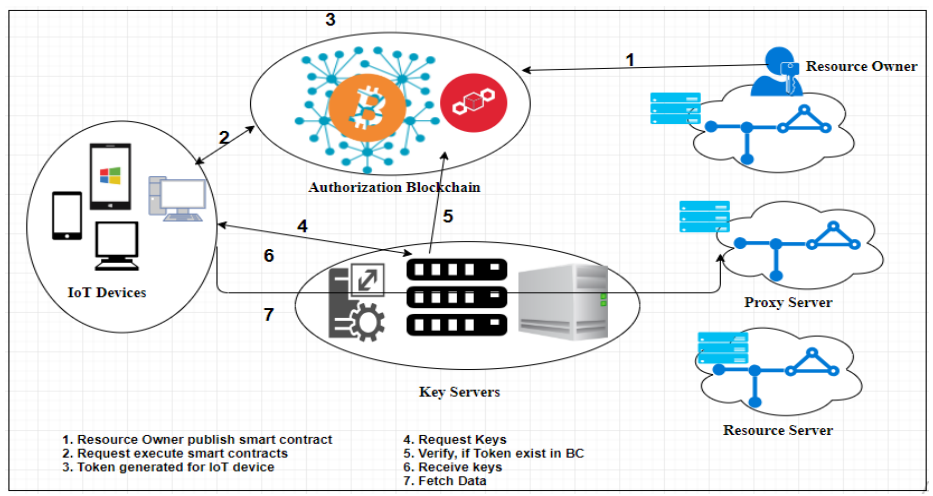

Figure 4 : IoT Chain framework

The authors explored the cloud and the MANN framework to connect smart devices to the internet of objects and provide communication security and represent an excellent framework called an internet-cloud framework, it is a good idea to provide secure connections to IoT devices. The authors provide a framework for middleware in the construction of MANET cloud access to data between IoT devices. In this article, a positive study on blockchains and IoT was conducted by researchers. They introduced the security concept to BC-IoT to improve IoT applications with the power of BCs.

\section{THE ROLE OF BC IN IoT}

IoT enables visual cables to exchange their data over a different network. IoT can be divided into the following categories.

1. Physical Objects: IoT provides a unique id of each object connected to the network. Material is able to exchange data with other IoT nodes.

2. Gates: Device gates work between material and cloud to ensure that communication is established, and security is provided by the network.

3. Connectivity: used to control data flow and establish a very short route between IoT sites.

4. Cloud: Used to store and count data.

$\mathrm{BC}$ is a series of verified blocks and those of encrypted encryption held by a network-connected device. Block data is stored in a publicly shared and distributed digital ledge. BC provides secure connection to the IoT network. A blockchain can be a private, public or consortium with a variety of structures. The following table represents the differences between all types of blockchains.

Table 1 : Kinds of Blockchains and their properties

\begin{tabular}{|c|c|c|c|c|c|c|}
\hline 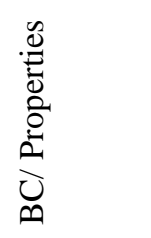 & 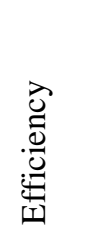 & 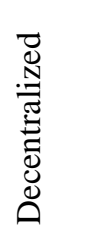 & 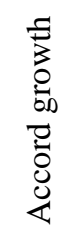 & 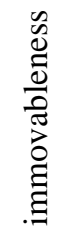 & 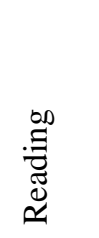 & 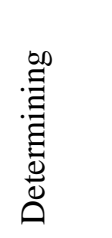 \\
\hline $\begin{array}{l}\text { Private } \\
\text { BC }\end{array}$ & $\begin{array}{l}\text { go } \\
\text { od }\end{array}$ & No & $e^{y}$ & $\begin{array}{l}\mathrm{C} \\
\text { an }\end{array}$ & $\begin{array}{r}\mathrm{Ca} \\
\mathrm{n} \quad \text { be }\end{array}$ & $\begin{array}{l}\text { Only } \\
\text { one }\end{array}$ \\
\hline
\end{tabular}




\begin{tabular}{|c|c|c|c|c|c|c|}
\hline & & & & be & $\begin{array}{l}\text { publi } \\
\text { cly }\end{array}$ & $\begin{array}{l}\text { industr } \\
\mathrm{y}\end{array}$ \\
\hline $\begin{array}{l}\text { Public } \\
\text { BC }\end{array}$ & rse $^{\text {wo }}$ & ${ }_{\mathrm{s}} \mathrm{Ye}$ & $0^{n}$ & $\mathrm{o}^{\mathrm{N}}$ & $\begin{array}{l}\text { pu } \\
\text { blicl } \\
\text { y }\end{array}$ & $\begin{array}{c}\text { All } \\
\text { miners }\end{array}$ \\
\hline $\begin{array}{r}\text { Conso } \\
\text { rtium BC }\end{array}$ & $\begin{array}{l}\text { go } \\
\text { od }\end{array}$ & $\begin{array}{l}\text { So } \\
\text { meti } \\
\text { mes }\end{array}$ & 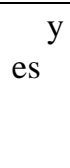 & $\begin{array}{l}\text { C } \\
\text { an } \\
\text { be }\end{array}$ & $\begin{array}{l}\mathrm{Ca} \\
\mathrm{n} \quad \text { be } \\
\text { publi } \\
\text { cly }\end{array}$ & $\begin{array}{c}\text { IoT } \\
\text { devices }\end{array}$ \\
\hline
\end{tabular}

The database in blockchains has features such as low-level reliability model, high security, public access, low-level privacy and transferable ownership when placed on a single database, properties are moderately trusted, low security, low public access, high privacy privacy and non-transferable. From the above structures, the blockchain is much more advanced than central storage.

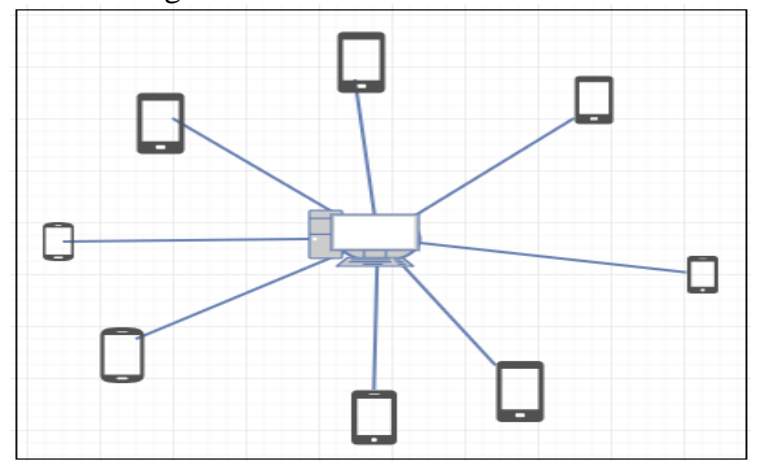

(a)

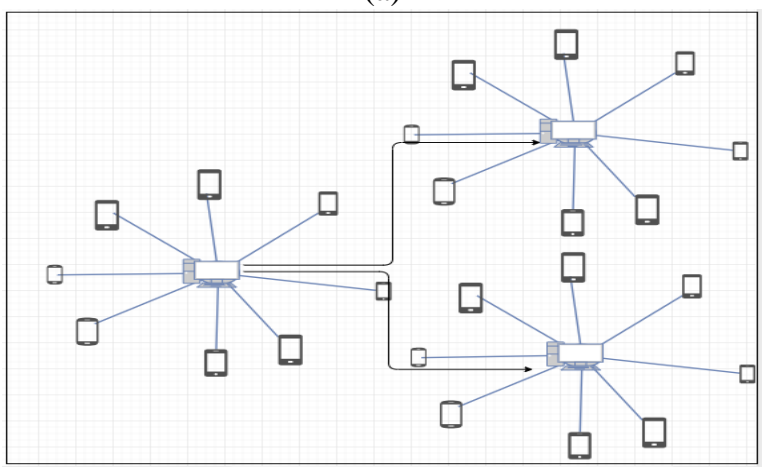

(b)

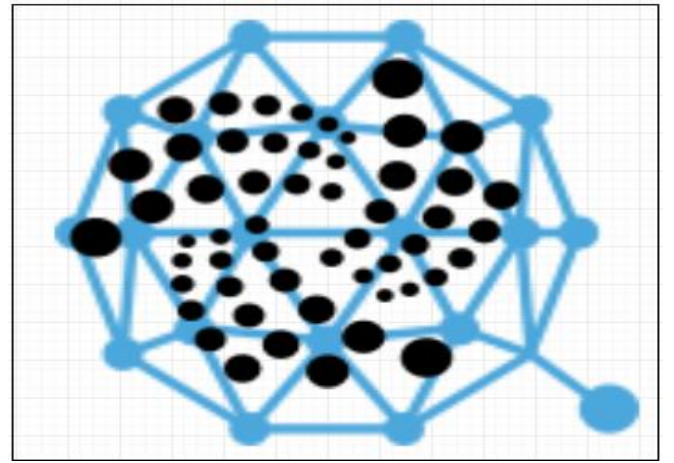

(c)

Figure 5 : (a) Centralized (b) Decentralized (c) Distributed

The following platforms are used to develop IoT systems using blockchain technology. a. IOTA: IOTA is a new blockchain and IoT platform called Next generation blockchains. This platform helps with high data integrity, high transaction performance and high blockchain performance through a few resources. It solves the limitations of blockchains.

b. IOTIFY: Provides an online web solution solution to reduce blockchains technology limitations in the form of custom applications.

c. Exec: An open source blockchain based tool. It helps your applications to the benefits of the cloud used.

d. Xage: It is a secure IoT blockchain platform for adding automation and secure information.

e. SONM: It is a medium-sized fog computing platform to provide secure cloud services.

IoTs and blockchains increase business opportunities and open up new markets where everyone or anything can communicate in real time with authenticity, privacy and security in the way they are used. The integration of these novel technologies will change the current world in which devices will communicate without people in various stages. The purpose of the framework is to obtain secure information in the right place, in the correct format, in real time. $\mathrm{BC}$ can be used to track billions of IoT connected objects, to link these objects, to enable transaction processing, to solve or eliminate failures and to create a flexible ecosystem to use the material in it. Hashing techniques used in data blocks by $\mathrm{BC}$ to create information privacy for users.

Nowadays, around the world smart devices are growing rapidly. They offer a lot of services to end users and attach to their daily lives. Mobiles currently use a mid-range mobile network for personal communication over the past decade. The smart phone is technically built to make the phone more usable for end users. We are now able to send text, photos, voice and video to each other using strong mobile networks. The smart phone can also connect to the internet easily by sending and receiving data within the mobile network. The Internet of Things describes a network of intelligent objects through which they can communicate and share information with each other using the Internet. Smart stuff with smart devices with built-in software, sensor and programs. Everything smart has a unique identifier on the network with their internal systems. Figure 1 shows that the smart device network network is a combination of intelligent device applications and an integrated framework installed by IEEE 802.15.4.

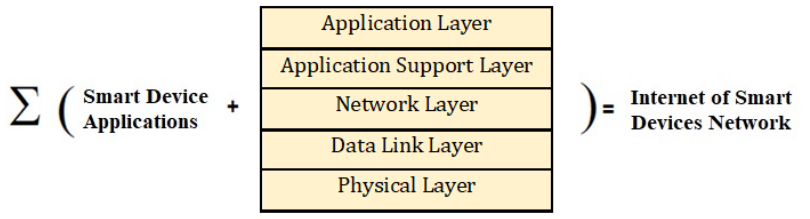

Fig.6: Internet of smart device network

Reliability is a major problem in connected areas where many sensors, actuators, controllers and smart devices etc are connected. Smart devices are not just smart phones, it can be a smart refrigerator, Smart home entry, smart air conditioners, Smart hubs, Smart thermostat, Smart LED converter Colors, Smart Watches and smart tablets etc. . connected to each other 
via the internet. The proposed research program creates a study that increases communication reliability on smart devices using IEEE802.15.4. Transferring data from one configuration to another using wireless networks dates back to 1973 in the form of radio network packets. They were able to communicate with other similar configuration devices. Recent work is underway on a project called Serval Project. Provides networking location for Android communication devices on a sub-network. While our research is concerned about the reliable connection to the internet of smart devices. The main contribution of this study is the construction of a communication framework and provided reliable communication using IEEE802.15.4 between the internet of smart devices. Previous studies have focused on building and utilizing a communication framework, but such research does not create a complete framework for reliable communication between the internet of smart devices. Figure 2 represents an IoT node with a reliability feature.

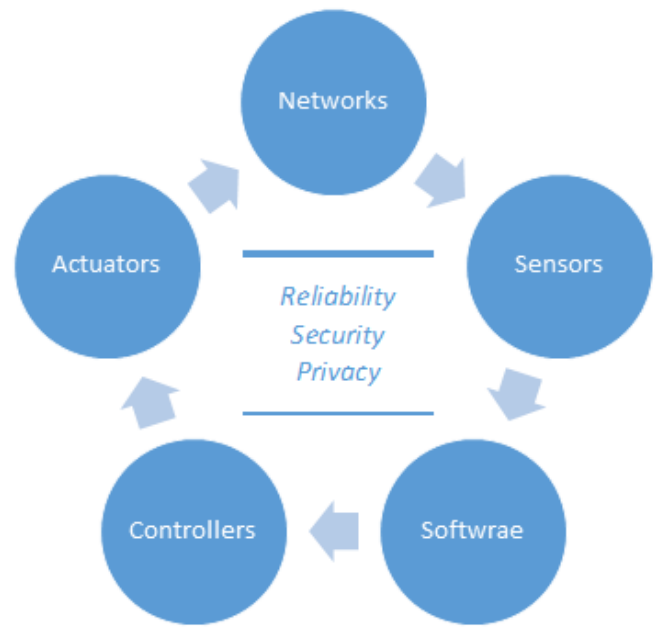

Fig. 7: IoT node with reliability

Integrity is a major problem in a variety of environments where many sensors, actuators, controllers and smart devices etc are connected to each other. The proposed study planned to build a study to increase communication reliability on devices using IEEE802.15.4. The main contribution of this study is the construction of a communication framework and provided reliable communication using IEEE802.15.4 between the internet of smart devices. Previous studies have focused on building and utilizing a communication framework, but such research does not create a complete framework for reliable communication between the internet of smart devices. The proposed online framework for smart devices based on IEEE 802.15.4 for reliable communication to improve the reliability of communication is tested and obtained positive results. The proposed study focuses on a framework for providing reliable internet connection to smart devices networks. Our main contribution to this study includes the reliability of the online communication framework for smart devices. This tutorial is very useful for the Internet of Things. The proposed framework was used for testing. Properly predicted in our full review. The overall effectiveness of the proposed device-based study device delays and communication reliability are assessed.

\section{OPPORTUNITIES}

The BC-IoT integration method has many amazing possibilities. It opens new doors for both of them together. Other opportunities are described as follows.

1. Building Trust between Groups: The BC-IoT approach will build trust between various connected devices due to its security features. Only verified devices can connect to the network and all transaction blocks will first be verified by miners so they can enter BC.

2. Reduce Costs: This method will reduce costs because it communicates directly without a third party. It removes all third-party nodes between sender and receiver. Provides direct communication.

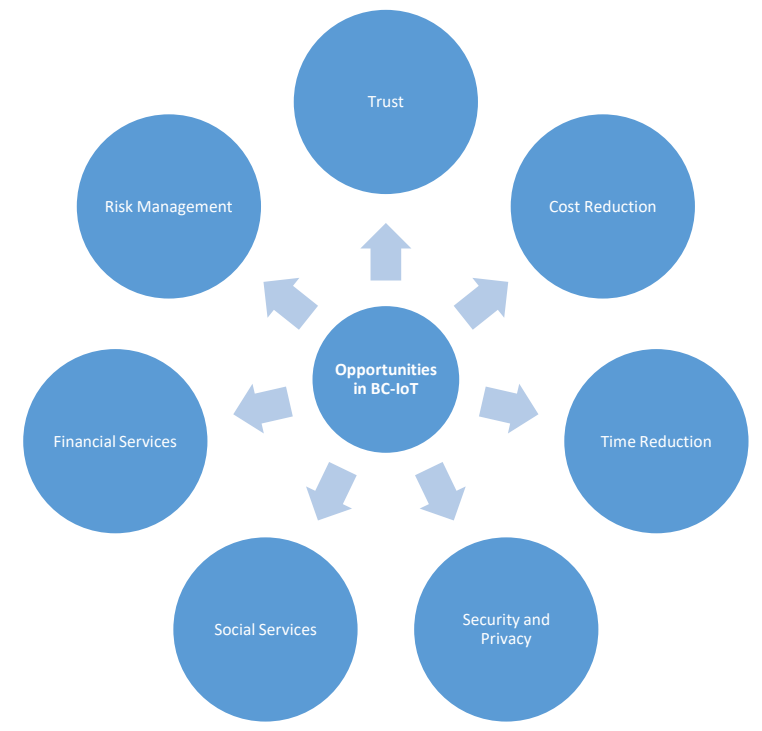

Figure 16 : Opportunities in BC-IoT

3: Reduce Time: This method greatly reduces time. Reduces transaction time taken from one day to two.

4: Security \& Privacy: Provides security and privacy to devices and information.

5. Social Services: This approach provides social and social services to connected devices. All connected devices are able to communicate and share information between them.

6. Financial Services: This method transfers funds securely without a third party. Provides fast, secure and independent financial services. Reduce costs and time.

7. Risk management: This approach plays a key role in analysing and mitigating the risk of resource failure and transactions.

\section{CHALLENGES}

IoT and $\mathrm{BC}$ can face many challenges such as scale, store, skills, acquisition and the following are some of the challenges facing the integration approach.

1. Diversity: BC can be suspended due to its heavy transaction load. Bitcoin storage exceeds 197 GB storage in 2019. Imagine 
if IoT meets BC the load will be much heavier than the current situation.

2. Storage: The digital bag will be stored on all IoT nodes. At the same time, it will increase with its storage size which will be a challenging task and become a heavy load on each connected device.

3. Lack of skills: BC new technology. It is known by very few people in the world. Therefore, it is also a challenge to train people professionally.

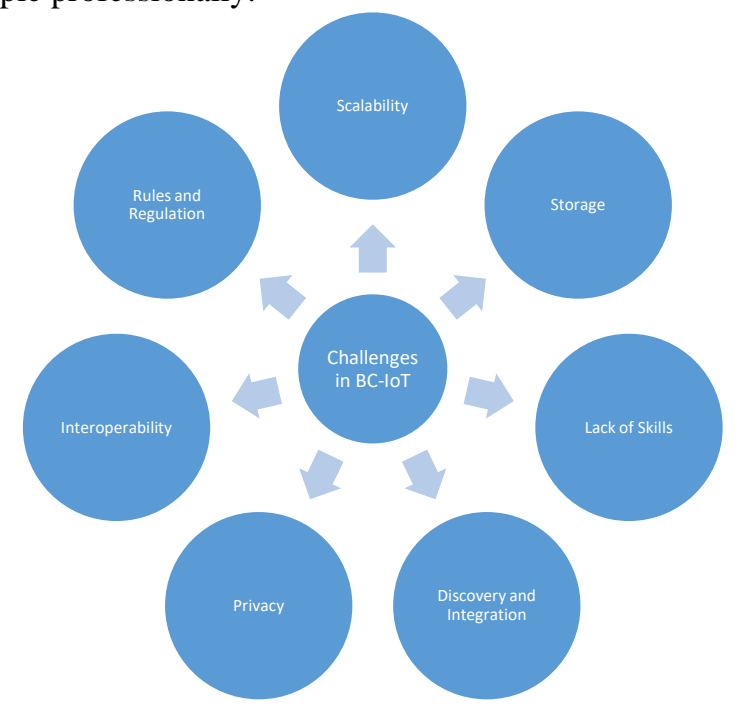

Figure 17 : Challenges in BC-IoT

Discovery and Integration: In fact, BC was not built for IoT. It is a very challenging task for connected devices to find another device in BC and IoT. Therefore, IoT nodes can detect but can detect and integrate $\mathrm{BC}$ with another device.

5. Privacy: The ledger is publicly distributed across all connected nodes. They see a ledger transaction. Therefore, privacy is a challenging task in an integrated approach.

6. Collaboration: BC can be public or private. Therefore, the interaction between public and private blockchains is also a challenge in the BC-IoT approach.

7. Rules and Regulations: IoT-BC will operate globally, and therefore deals with a number of rules and regulations for the use of this method worldwide.

\section{CONCLUSION}

$\mathrm{BC}$ and IoT are the novels tested in this document. Many opportunities and challenges are described. Also, the available platforms are listed in this article. This approach could be the future of the internet because it can transform the current internet system and transform it into a new one where all smart devices will connect to other devices using a peer-to-peer network in real time. It can reduce costs and current time and provide relevant information to the right device in real time. Therefore, it can be very helpful in the future.

\section{REFERENCES}

[1] Alam, Tanweer, and Mohammed Aljohani. "Design a new middleware for communication in ad hoc network of android smart devices." In Proceedings of the Second International Conference on Information and Communication Technology for Competitive Strategies, pp. 1-6. 2016. https://doi.org/10.1145/2905055.2905244

[2] Aljohani, Mohammed, and Tanweer Alam. "An algorithm for accessing traffic database using wireless technologies." In 2015 IEEE International Conference on Computational Intelligence and Computing Research (ICCIC), pp. 1-4. IEEE, 2015. https://doi.org/10.1109/ICCIC.2015.7435818

[3] Aljohani, Mohammed, and Tanweer Alam. "Design an Mlearning framework for smart learning in ad hoc network of Android devices." In 2015 IEEE International Conference on Computational Intelligence and Computing Research (ICCIC), pp. $1-5 . \quad$ IEEE, 2015. https://doi.org/10.1109/ICCIC.2015.7435817

[4] Alam, Tanweer, and Mohammed Aljohani. "An approach to secure communication in mobile ad-hoc networks of Android devices." In 2015 International Conference on Intelligent Informatics and Biomedical Sciences (ICIIBMS), pp. 371-375. IEEE, 2015. https://doi.org/10.1109/ICIIBMS.2015.7439466

[5] Alam, Tanweer, and Mohammed Aljohani. "Design and implementation of an Ad Hoc Network among Android smart devices." In 2015 International Conference on Green Computing and Internet of Things (ICGCIoT), pp. 1322-1327. IEEE, 2015. https://doi.org/10.1109/ICGCIoT.2015.7380671

[6] Alam, Tanweer. "Internet of things and blockchain-based framework for coronavirus (covid-19) disease." Available at SSRN $3660503 \quad$ (2020). https://doi.org/10.22541/au.159569226.68851353

[7] Alam, Tanweer. "Blockchain-Enabled Mobile Healthcare System Architecture for the Real-Time Monitoring of the COVID-19 Patients." Available at SSRN 3772643 (2021). https://doi.org/10.2139/ssrn.3772643

[8] Alam, Tanweer. "IoT-Fog-Blockchain Framework: Opportunities and Challenges." International Journal of Fog $\begin{array}{lllll}\text { Computing (IJFC) } 3, & \text { no. } 2 & \text { (2020): } & 1-20 \text {. }\end{array}$ https://doi.org/10.4018/IJFC.2020070101

[9] Alam, Tanweer, and Mohammed Aljohani. "Software Defined Networks: Review and Architecture." Alam, T., \& Aljohani, M.(2020). Software Defined Networks: Review and Architecture. IAIC Transactions on Sustainable Digital Innovation (ITSDI) 1, no. 2 (2020): 143-151. https://doi.org/10.34306/itsdi.v1i2.114

[10] Alam, Tanweer. "Device-to-Device Communications in Cloud, MANET and Internet of Things Integrated 
Architecture." Journal of Information Systems Engineering and Business Intelligence. doi 10 (2020): 18-26. https://doi.org/10.20473/jisebi.6.1.18-26

[11] Rababah, Baha, Tanweer Alam, and Rasit Eskicioglu. "The Next Generation Internet of Things Architecture Towards Distributed Intelligence: Reviews, Applications, and Research Challenges." Journal of Telecommunication, Electronic and Computer Engineering (JTEC) 12, no. 2 (2020). https://doi.org/10.36227/techrxiv.12657182.v2

[12] Tanweer Alam. Abdulrahman A. Salem. Ahmad O. Alsharif. Abdulaziz M. Alhujaili." Smart Home Automation Towards the Development of Smart Cities.", Computer Science and Information Technologies 1, no. 1 (2020). https://doi.org/10.34306/csit.v5i1.119

[13] Alam, Tanweer, Baha Rababah, Arshad Ali, and Shamimul Qamar. "Distributed Intelligence at the Edge on IoT Networks." Annals of Emerging Technologies in Computing $\begin{array}{llllll}(\mathrm{AETiC}) & 4 & \text { no. } & 5 & \text { (2020): } & 1-18 .\end{array}$ https://doi.org/10.33166/AETiC.2020.05.001

[14] Alam, Tanweer, Abdirahman Ahmed Hadi, Rayyan Qari Shahabuddin Najam, and Shamimul Qamar. "Design a Mobile Application for Children's Tracking in Crowded Environments." TEST Engineering and Management 83 (2020). https://doi.org/10.36227/techrxiv.12750848

[15] Alam, Tanweer. "Performance evaluation of blockchains in the internet of things." Computer Science and Information Technologies 1, no. 3 (2020): 93-97. https://doi.org/10.11591/csit.v1i3.p93-97

[16] Alam, Tanweer. "CMI Computing: A Cloud, MANET and Internet of Things Integration for Future Internet.", JAMBURA JOURNAL OF INFORMATICS 2, no. 1 (2020). https://doi.org/10.37905/jii.v2i1.4539

[17] Alam, Tanweer, and Mohammed Aljohani. "M-Learning: Positioning the Academics to the Smart devices in the Connected Future." JOIV: International Journal on Informatics Visualization 4, no. 2 (2020): 76-79. https://doi.org/10.30630/joiv.4.2.347

[18] Alam, Tanweer. "Coronavirus disease (COVID-19): reviews, applications, and current status." Applications, and Current Status (July 25, 2020) (2020). https://doi.org/10.32493/informatika.v5i3.6563

[19] Tanweer Alam. Yazeed Mohammed Alharbi. Firas Adel Abusallama. Ahmad Osama Hakeem. "Smart Campus Mobile Application Toward the Development of Smart Cities.", International Journal of Applied Sciences and Smart Technologies. $\quad$ Vol2 $\quad 1 \quad$ (2020). https://doi.org/10.2139/ssrn.3638973
[20] Alam, Tanweer. "Internet of Things: A Secure CloudBased MANET Mobility Model.", International Journal of Network Security 22, no. 3 (2020). https://doi.org/10.31219/osf.io/py6ak

[21] Alam, Tanweer. "Design a blockchain-based middleware layer in the Internet of Things Architecture." JOIV: International Journal on Informatics Visualization 4, no. 1 (2020): 28-31. https://doi.org/10.30630/joiv.4.1.334

[22] Alam, Tanweer. "mHealth Communication Framework using blockchain and IoT Technologies." International Journal of Scientific \& Technology Research 9, no. 6 (2020). https://doi.org/10.31219/osf.io/byvpu

[23] Alam, Tanweer, and Mohammed Aljohani. "Decision support system for real-time people counting in a crowded environment." IJ of Electronics and Information Engineering 12, no. 11 (2020): $34-41$. https://doi.org/10.2139/ssrn.3639076

[24] Alam, Tanweer. "Cloud Computing and its role in the Information Technology." Tanweer Alam.(2020). Cloud Computing and its role in the Information Technology. IAIC Transactions on Sustainable Digital Innovation (ITSDI) 1, no. 2 (2020): 108-115. https://doi.org/10.34306/itsdi.v1i2.103

[25] Alam, Tanweer. "Efficient and secure data transmission approach in cloud-MANET-IoT integrated framework.", Journal of Telecommunication, Electronic and Computer Engineering $12, \quad$ no. 1 (2020).. https://doi.org/10.22541/au.159164756.64416520

[26] Alam, Tanweer. "Tactile internet and its contribution in the development of smart cities." International Journal of Electronics and Information Engineering 12, no. 3 (2020): 112-118. https://doi.org/10.6636/IJEIE.202009_12(3).02

[27] Alam, Tanweer. "Cloud-MANET and its Role in Software-Defined Networking." Transactions on Science and $\begin{array}{llllll}\text { Technology } 7, \quad \text { no. } & 1 & \text { (2020): } & 1-7 .\end{array}$ https://doi.org/10.31219/osf.io/e536q

[28] Tanweer Alam., "Middleware implementation in MANET of Android Devices.", International Journal of Electronics and Information Engineering 12, no. 2 (2020). https://doi.org/10.2139/ssrn.3638977

[29] Alam, Tanweer, Shamimul Qamar, Amit Dixit, and Mohamed Benaida. "Genetic Algorithm: Reviews, Implementations, and Applications." International Journal of Engineering Pedagogy https://doi.org/10.3991/ijep.v10i6.14567
(2020). 
[30] Alam, Tanweer, and Baha Rababah. "Convergence of MANET in communication among smart devices in IoT.", International Journal of Wireless and Microwave Technologies $\quad$ (IJWMT), $\quad 9: \quad 2$. https://doi.org/10.5815/ijwmt.2019.02.01

[31] Alam, Tanweer. "5G-Enabled Tactile Internet for smart cities: vision, recent developments, and challenges." Jurnal Informatika 13, no. 2 (2019): 1-10. https://doi.org/10.26555/jifo.v13i2.a13426

[32] Alam, Tanweer. "A middleware framework between mobility and IoT using IEEE 802.15. 4e Sensor Networks." Jurnal Online Informatika 4, no. 2 (2020): 90-94. https://doi.org/10.15575/join.v4i2.487

[33] Alam, Tanweer. "Blockchain and its Role in the Internet of Things (IoT).", International Journal of Scientific Research in Computer Science, Engineering and Information $\begin{array}{lllll}\text { Technology } & 5, & \text { no. } & 1 & \text { (2019). }\end{array}$ https://doi.org/10.32628/CSEIT195137

[34] Alam, Tanweer. "IoT-Fog: A communication framework using blockchain in the internet of things.", International Journal of Recent Technology and Engineering (IJRTE) 7, no. 6 (2019). https://doi.org/10.36227/techrxiv.12657200

[35] Alam, Tanweer. "A reliable framework for communication in internet of smart devices using IEEE 802.15. 4.", ARPN Journal of Engineering and Applied Sciences 13 : 10. https://doi.org/10.22541/au.159164762.21634659

[36] Alam, Tanweer. "A reliable communication framework and its use in internet of things (IoT)." CSEIT1835111| Received 10 (2018): 450-456.

[37] Alam, Tanweer, and Mohamed Benaida. "The role of cloud-MANET framework in the internet of things (IoT).", International Journal of Online and Biomedical Engineering 14: 12, 2019. https://doi.org/10.3991/ijoe.v14i12.8338

[38] Tanweer Alam and Mohamed Benaida., "CICS: CloudInternet Communication Security Framework for the Internet of Smart Devices.", International Journal of Interactive Mobile Technologies (iJIM) 12, no. 6 (2018). https://doi.org/10.3991/ijim.v12i6.6776

[39] Alam, Tanweer. "Middleware implementation in cloudMANET mobility model for internet of smart devices.", International Journal of Computer Science and Information Security, 17: 5. 86-94. https://doi.org/10.2139/ssrn.3638980

[40] Alam, Tanweer. "Fuzzy Control Based Mobility Framework for Evaluating Mobility Models in MANET of
Smart Devices.", ARPN Journal of Engineering and Applied Sciences 12: 15 .

[41] Alam, Tanweer, and Mohamed Benaida. "Blockchain, Fog and IoT Integrated Framework: Review, Architecture and Evaluation.", Technology Reports of Kansai University 62, no. 2 (2020). https://doi.org/10.31219/osf.io/8k7j9

[42] Sharma, Abhilash, Tanweer Alam, and Dimpi Srivastava. "Ad hoc network architecture based on mobile Ipv6 development.", Advances in Computer Vision and Information Technology, 224 (2008).

[43] Alam, Tanweer. "Blockchain-based Big Data Analytics Approach for Smart Cities." Authorea Preprints (2020). https://doi.org/10.22541/au.160208029.93138178/v1

[44] Alam, Tanweer, and B. K. Sharma. "A new optimistic mobility model for mobile ad hoc Networks." International Journal of Computer Applications 8, no. 3 (2010): 1-4. https://doi.org/10.5120/1196-1687

[45] Alam, Tanweer, Parveen Kumar, and Prabhakar Singh. "Searching mobile nodes using modified column mobility model." International Journal of Computer Science and Mobile Computing 3, no. 1 (2014): 513-518.

[46] Alam, Tanweer, and Mohamed Benaida. "Blockchain and Internet of Things in Higher Education.", Universal Journal of Educational Research., 8(5): 2164-2174, 2020. DOI: 10.13189/ujer.2020.080556.

https://doi.org/10.13189/ujer.2020.080556

[47] Alam, Tanweer, and Moath Erqsous., "The Real-Time Alert System for Prayers at Smart Masjid.", Scientific Journal of Informatics, 7, no. 2 (2020): 166-172.

[48] Singh, Parbhakar, Parveen Kumar, and Tanweer Alam. "Generating different mobility scenarios in ad hoc networks.", International Journal of Electronics Communication and Computer Technology, 4, no. 2 (2014): 582-591. 